\title{
Surgical Management of Cervical Tracheal Stenosis
}

\author{
Rokade Vidya, Lecturer, Dehadray A.Y, Associate Professor \\ Dept. of ENT, Sassoon General Hospital, Pune.
}

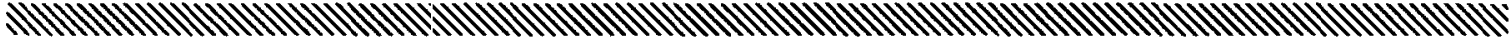

\section{Abstract}

Postintubation cervical tracheal stenosis managed by tracheal resection with supralaryngeal release and end to end ancstomosis, without postoperative stent or endotracheal tube.

Cervical tracheal stenosis of $<3 \mathrm{~cm}$ in length can be managed by E.N.T. surgeon alone.

\section{INTRODUCTION}

aryngotracheal stenosis is a complex prob - lem for which multiple divergent and radically different reconstructive options are available. The precise method of reconstruction chosen for a given lesion must be based on sound judgement to maximise the chances for successful outcome and minimise the potential for making the stenosis worse.

The commonest cause for tracheal stenosis is prolonged intubation with cuffed end tracheal tube. The cuff of the endotracheal tube will produce circumferential pressure injury in the trachea and subsequently the stenosis in the trachea. If erosion has been deep and has destroyed the cartilages the resulting stenosis will under no circumstances be amenable to cure by conservative method.

\section{Case Report}

A 30 year old tracheostomised male patient attended the E.N.T. Outpatient department in March 1997. His main complaint was discomfort and tightness in chest, total loss of laryngeal speech. ICL examination showed normal vocal cord movements. X-ray chest showed the broken metal tracheostomy tube in right main bronchus. F.B. was removed through tracheostome under general anaesthesia. During bronchoscopy we found the circumferential tracheal stenosis above tracheostomy site with just pinhole inner -diameter.
On detail 1 enquiry we found that in 1993 because of snake bite he was admitted in I.C.U. He was on ventilator with endotracheal tube for ten days. After that he was discharged from the hospital and was alright for weeks. Then subsequently patient became breathless for which tracheostomy was done.

$X$-ray neck lateral view showed the tracheal stenosis abovt the level of the tracheostomy (Fig.1).

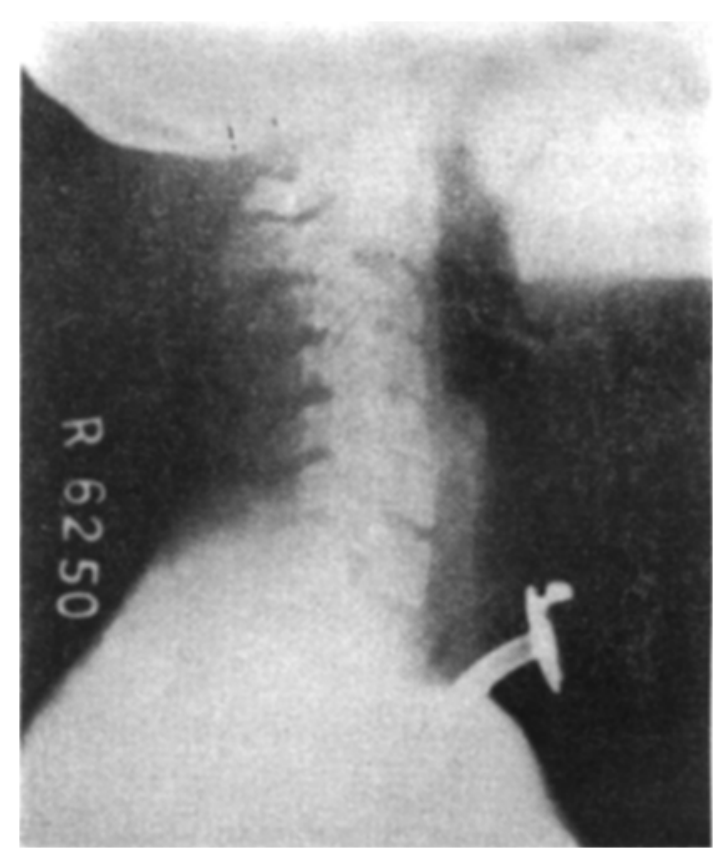

Fig 1 : X-ray neck lat view, showing tracteat stenotic orea 
CT Scan (Fig. 2 and Fig.3): Stenosis in cervical trachea above the level of tracheostomy. Total length of narrowing was $2.75 \mathrm{~cm}$ proximal to tracheostomy site and $1 \mathrm{~cm}$ distal to cricoid cartilage. Maximum narrowing was noted at distance about $2.75 \mathrm{~cm}$ proximal to tracheostomy site and the inner diameter of trachea as that site was $2 \mathrm{~mm} \times 2 \mathrm{~mm}$. Larynx and laryngeal cartilages were normal. Trachea distal to tracheostomy site was normal.

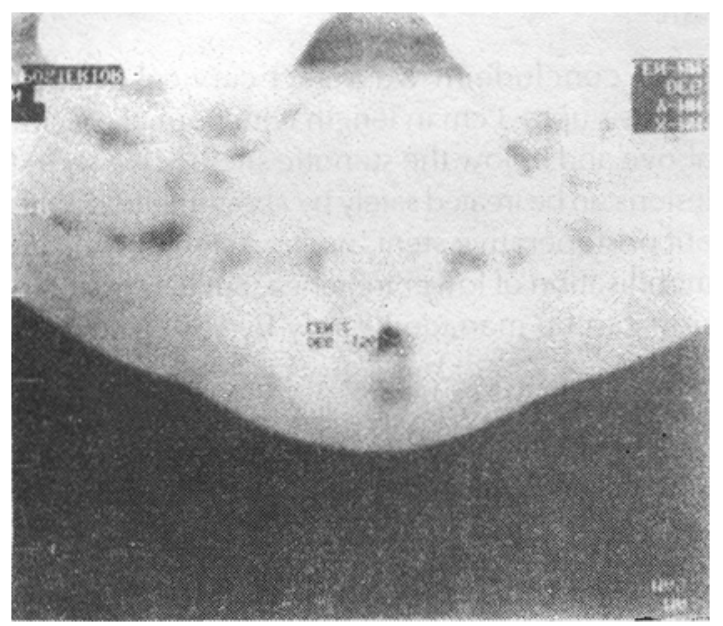

Fig 2: C. T. Scan, showing the tracheal stenosis \& inner dia $2 \times 2 \mathrm{~mm}$

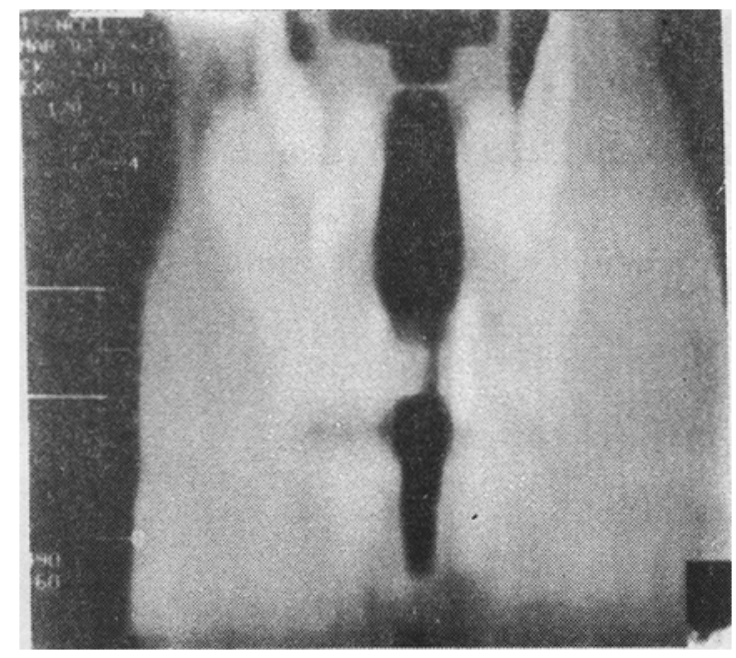

Fig 3 : C. T. Scan, showing the stenotic area

\section{Surgical Procedure:}

Tracheal resection with supralaryngeal release under general anaesthesia.

Sorensen's incisoin was made ' $U$ ' shape flap elevated upto hyoid bone. Deep fascia was incised in midline. The isthmus of the thyroid gland was transected. The whole of the cervical trachea was exposed upto the sternal notch. The stenotic area was exposed, and transected. Then both the thyrohy muscles transected. The superior cornu of the thyroid oid cartilage exposed and trasected. The thyrohyoid membrane was transected near to the superior edge of the thyroid cartilage with care being taken not to injury the internal branch of superior laryngeal nerve. End to end anastomosis of the trachea was done by using 3/ 0 prolene and keeping the knot outside the lumen. Tracheostomy was kept as it is. Stent was not used. Wound was sutured with $3 / 0$ blck silk. Neck was kept in flexed position by taking a stitch from chin to chest and by a well placed posterior cervical moulded plaster splint. Postoperative period was uneventful. Flexion of the neck was

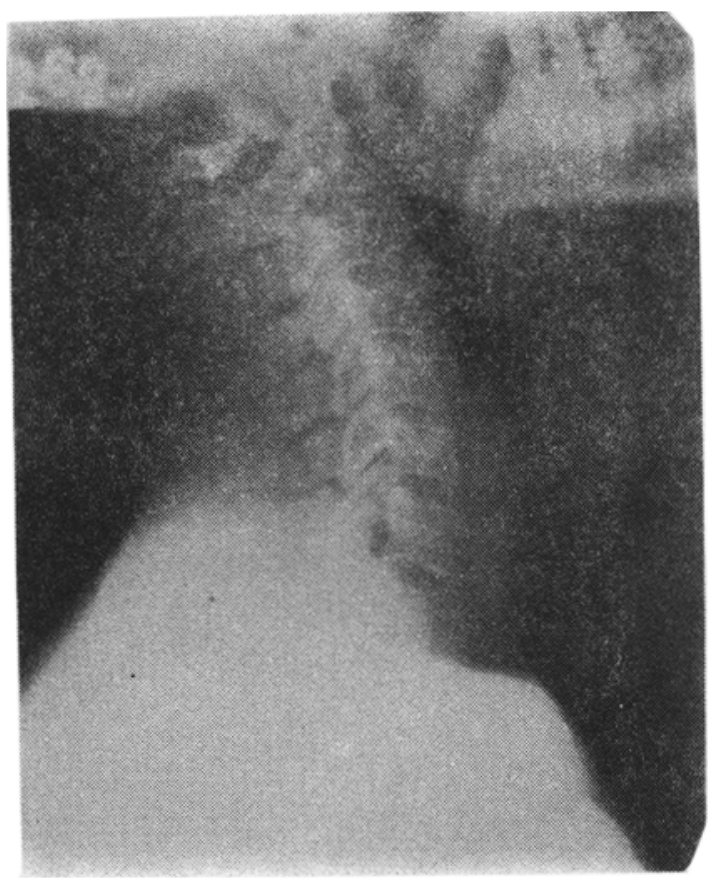

Fig 4 : Post operative, $X$-ray neck lat view 
maintained for ten days. Tracheostomy closure was done after one month. Patient has developed normal laryngeal speech and normal nasal breathing pattern (Fig. 4).

\section{Discussion}

Prolonged endotracheal intubation with cuffed endotracheal tube is the commonest cause for tracheal stenosis. The cuff of the endotracheal tube is $2.5 \mathrm{~cm}$ in length, area which comes in contact with mucosa is $1.75 \mathrm{~cm} \pm 0.5 \mathrm{~cm}$. So in majority of the cases stenotic area will not be more than 2.5 $\mathrm{cm}$. Laryngeal drop surgery gives 2.5 to $3 \mathrm{~cm}$ additional length for reconstruction by end to end anastomosis. There is no need of risky procedures like sternal splitting and mobilisation of the lower trachea. The mobilisation of lower trachea gives pull on suture line because of negative intrathorasic pressure, with subsequent chances of complica- tions.

Postoperative endotracheal intubation or stent at the resection area is extremely uncomfortable and again it will predispose the chances of tracheal stenosis. Postoperative stent or endotracheal intubation is a standard procedure recommended by the standard textbooks (Paparella, Lore etc). We can manage the patient safely in immediate postoperative period with tracheostomy, afterwards patient can be decanulated without any problem.

So to conclude, if we detect cervical tracheal stenosis upto $3 \mathrm{~cm}$ in length with normal mucosa above and below the stenotic site, such a type of lesion can be treated safely by above method, without postoperative stent, without sternal split and mobilisation of lower treachea. Such a type of lesion can be managed safely by E.N.T. surgeon alone.

\section{References}

1. Hermes C.Grillo, M.D., Douglas J. Mathisen, M.D., (1991): Diseases of the trachea and bronchi. Otolaryngology (Vol.III). Paparella W.B. Saunders Co., pg 2385 to 2396.

2. J. Paul Willging and Robin J. Cotton (1995): Reconstruction of laryngotracheal stenosis in the adult : Advances in Otolaryngology. Head and neck Surgery, Vol.9: Mosby, pg 49 to 65.

3. Lore (1988): An Atlas of Head and Neck Surgery. Third edition, W.B. Saunders Co. pg 820 to 827.

4. A.G.D. Maran (1987): Trauma and stenosis of the larynx. Scott Brown's Otolaryngology. Fifth edition, Butterworth, Jaypee Brothers, pg 153 to 154.

5. Stell and Maran's (199.4): Head and Neck Surgery. Third edition, Butterworth, Heinemann, pg 156 to 157. 